\title{
Electric field gradients and impurity distributions in doped noble metals: A systematic study
}

\author{
I. J. R. Baumvol, M. Behar, ${ }^{*}$ J. A. H. da Jornada, R. P. Livi, K. W. Lodge, A. Lopez Garcia, ${ }^{\dagger}$ and F. C. Zawislak \\ Instituto de Física, Universidade Federal do Rio Grande do Sul. 90000 Porto Alegre. Brasil
}

(Received 6 August 1979)

\begin{abstract}
The electric field gradients created at ${ }^{111} \mathrm{Cd}$ nuclei by dilute transition-element impurities in noble metals are studied by the technique of time-differential perturbed angular correlation. The present results supplement previous ones with data for the alloys $C u \mathrm{Rh}, C u \mathrm{Pt}, A g \mathrm{Rh}$, $A g \mathrm{Ir}, A u \mathrm{Rh}, A u \mathrm{Pd}$, and $A u \mathrm{Pt}$, including temperature and concentration dependence in some typical cases. The results show conclusively that, for most impurities situated to the left of the host noble metals in the Periodic Table, there is a strong attraction between the probe In and the impurity atoms. The binding energy $\Delta E_{B}$ for the probe-impurity system is measured for the $C u \mathrm{Rh}$ and $C u \mathrm{Pt}$ alloys. The temperature dependence of the high-frequency quadrupole interaction $v_{h}$ due to a single-impurity nearest neighbor to the probe is found to obey the usual empirical law $\nu(T)=\nu(0)\left(1-\alpha T^{3 / 2}\right)$. The wealth of experimental data thus made available allows a systematic examination of the results from which the following facts emerge: (i) The observed high frequency $\nu_{h}$ is related to the nominal valence difference $\Delta Z$ between impurity and host and to a parameter $\lambda$, the difference in the period of impurity and host in the Periodic Table; (ii) a correlation of $\Delta E_{B}$ with both $\Delta Z$ and $\lambda$ is apparent; and (iii) an inverse correlation between the $\alpha$ parameter and $\Delta E_{B}$ is apparent.
\end{abstract}

\section{INTRODUCTION}

In a series of papers we have reported investigations, using the technique of time-differential perturbed angular correlation (TDPAC), of the electric field gradients (EFG) created by different impurity atoms dissolved in the cubic noble metals of $\mathrm{Ag}^{1-3}$ and $\mathrm{Cu}^{4}$ One important result shown by this work is that for impurities located to the left of the Ag and $\mathrm{Cu}$ hosts in the Periodic Table, there is a strong enhancement of impurity population nearest neighbor (NN) to the ${ }^{111}$ In probe atoms. This enhancement, over a random distribution of $\mathrm{NN}$ impurities, occurs for the alloys of $A g \mathrm{Pd},{ }^{3} \mathrm{CuPd},{ }^{4}$ and $A g \mathrm{Pt}^{5,6}$ For the $A g$ Pd system with 0.25 at. $\%$ of $\mathrm{Pd}$, it was observed that $30 \%$ of the probe nuclei feel a high-frequency interaction instead of about $3 \%$ which would be expected from a random distribution of impurities. Aided by this strong enhancement we have investigated various other interesting aspects such as the mechanism of population of impurities around the probe atoms, the temperature dependence of the created EFG, dynamical processes at high temperatures and binding energies between impurity and probe atoms and these are reported in the above references.

It now seems important to extend these experiments to include other impurity atoms and also to use the cubic noble metal Au as a host. A systematic study can give additional information concerning the role of the host (the influence of its lattice and electronic structures) as well as the effect of impurities with different valences on the above-mentioned properties.

In this paper we report the results of the studies of the EFG generated by impurities of $\mathrm{Pt}$ and $\mathrm{Rh}$ in $\mathrm{Cu}$ host, $\mathrm{Rh}$ and $\mathrm{Ir}$ in $\mathrm{Ag}$ host, and $\mathrm{Pd}, \mathrm{Rh}$, and $\mathrm{Pt}$ in $\mathrm{Au}$ host. The temperature dependence of the EFG in the alloys of $A g \mathrm{Rh}, C u \mathrm{Rh}$, and $C u \mathrm{Pt}$ was also investigated. In one case, $A u \mathrm{Pd}$, the concentration of impurity was varied. Table I shows the alloys studied in this paper together with those previously investigated, and also gives the concentration of impurities.

In Sec. II we describe the sample preparation and the data treatment. Section III shows the obtained results. A discussion of the present and previous results is presented in Sec. IV. The most important conclusions are presented in Sec. V.

\section{EXPERIMENTAL PROCEDURE}

\section{A. Sample preparation}

The transition metals used as impurities in this work have high melting points compared to the host noble metals. Due to this the solid solutions were prepared in two steps. First a "mother alloy" of $c$ at. \% of the impurity $(0.3 \leqslant c \leqslant 8.0$, depending on the impurity solubility in the host ${ }^{7,8}$ ) with the noble metal was melted in an arc furnace. In a second step, adequate quantities of this alloy and the noble metal were melted in a resistance furnace. 
TABLE I. Summary of the investigated alloys (hosts and impurity concentrations).

\begin{tabular}{|c|c|c|c|}
\hline \multirow[b]{2}{*}{ Host } & \multirow[b]{2}{*}{ Impurity } & \multicolumn{2}{|c|}{ Measured impurity concentration } \\
\hline & & $\begin{array}{c}\text { Room temperature } \\
(\text { at. } \%)\end{array}$ & $\begin{array}{c}\text { Function of temperature } \\
(\text { at. } \%)\end{array}$ \\
\hline \multirow{3}{*}{$\mathrm{Cu}$} & $\mathrm{Pd}^{\mathrm{a}}$ & $0.5,1.0,1.5,2.5,4.5$ & 0.7 \\
\hline & $\mathrm{Pt}$ & 1.0 & 1.0 \\
\hline & $\mathrm{Rh}$ & 0.5 & 0.5 \\
\hline \multirow{5}{*}{$\mathrm{Ag}$} & $\mathrm{Pd}^{\mathrm{b}}$ & $0.25,0.5,1.0,1.5,2.0,2.5$ & 0.5 \\
\hline & $\mathrm{Rh}$ & $0.25,0.5$ & 0.25 \\
\hline & & & \\
\hline & $\mathrm{Ir}$ & 0.2 & \\
\hline & $\mathrm{Pt}^{\mathrm{c}}$ & $0.3,1.0$ & 0.3 \\
\hline \multirow{3}{*}{$\mathrm{Au}$} & $\mathrm{Pd}$ & $0.5,1.0,2.0,3.0,4.5$ & . \\
\hline & $\mathrm{Rh}$ & 0.5 & \\
\hline & $\mathrm{Pt}$ & 0.5 & \\
\hline
\end{tabular}

For the $\mathrm{Cu}$ and the Au alloys, the radioactive carrier-free ${ }^{111}$ In obtained through the reaction ${ }^{109} \mathrm{Ag}(\alpha, 2 n)^{111}$ In was electroplated on the mother alloy before the second step and for the Ag alloys we used the $\alpha$-irradiated $\mathrm{Ag}$ directly.

All the samples were melted in argon atmosphere, and annealed, also in argon, for some hours at $800{ }^{\circ} \mathrm{C}$. The purity of the component metals was $\geqslant 99.99 \%$. This means that our samples contain three elements, the host, the solute impurity atoms, and the very dilute carrier-free, radioactive atoms of ${ }^{111} \mathrm{In}$, which decay to ${ }^{111} \mathrm{Cd}$.

\section{B. Data treatment}

The TDPAC experiments were performed using the $173-247-\mathrm{keV} \gamma-\gamma$ cascade in ${ }^{111} \mathrm{Cd}$, to measure the hyperfine interaction of the nuclear quadrupole moment of the $247-\mathrm{keV}$ state with the EFG generated at the probe nucleus by the impurity.

The TDPAC data were analyzed in the usual way. ${ }^{9}$ From the measured coincidence counting rates at angles of $90^{\circ}$ and $180^{\circ}$, the ratio

$C(t)=2 \frac{C\left(180^{\circ}, t\right)-C\left(90^{\circ}, t\right)}{C\left(180^{\circ}, t\right)+2 C\left(90^{\circ}, t\right)}=A_{22} G_{22}(t)$

was calculated, after corrections for random coincidences and misalignment of the sample. The perturbation factor

$G_{22}(t)=\sigma_{20}+\sum_{n=1}^{3} \sigma_{2 n} \cos \left(\omega_{n} t\right) \exp \left(-\frac{1}{2} \delta \omega_{n} t\right)$ contains the information about the nuclear quadrupole interaction in the frequencies $\omega_{n}$. These specify the nuclear quadrupole frequency, $v_{Q}$, and the asymmetry parameter, $\boldsymbol{\eta}$,

$$
\nu_{Q}=e Q V_{z z} / h, \quad \eta=\left(V_{x x}-V_{y y}\right) / V_{z z} .
$$

Here $V_{z z}, V_{y y}$, and $V_{x x}$ are the usual principal components of the EFG, and $Q$ is the quadrupole moment of the $247-\mathrm{keV}$ state in ${ }^{111} \mathrm{Cd}$. The term $\exp \left(-\frac{1}{2} \delta \omega_{n} t\right)$ in expression (2) accounts for the Lorentzian distribution of frequencies having a width $\delta$.

In all cases it was observed that a part $\left(a_{h}\right)$ of the probe nuclei are subjected to high-frequency interactions $\nu_{h}$, a second part $\left(a_{l}\right)$ to a distribution of low frequencies centered around $\nu_{l}$, and a third part $\left(a_{0}\right)$ do not feel any hyperfine interaction. Adequate computer fittings were performed using expression (2) in order to extract the amplitudes and the corresponding frequencies from each $A_{22} G_{22}(t)$ curve.

\section{RESULTS}

Typical results for the perturbation factor $A_{22} G_{22}(t)$ are shown in Figs. 1-3. The full lines in the figures show the computed least-squares fittings. The parameters obtained from the fittings are displayed in Tables II-IV.

In particular, the results shown in Table II are for the lowest measured concentration of impurity in each alloy, including the data from our previous experiments for $A g \mathrm{Pd}$ (Ref. 3) and $C u \mathrm{Pd}$ (Ref. 4) as 


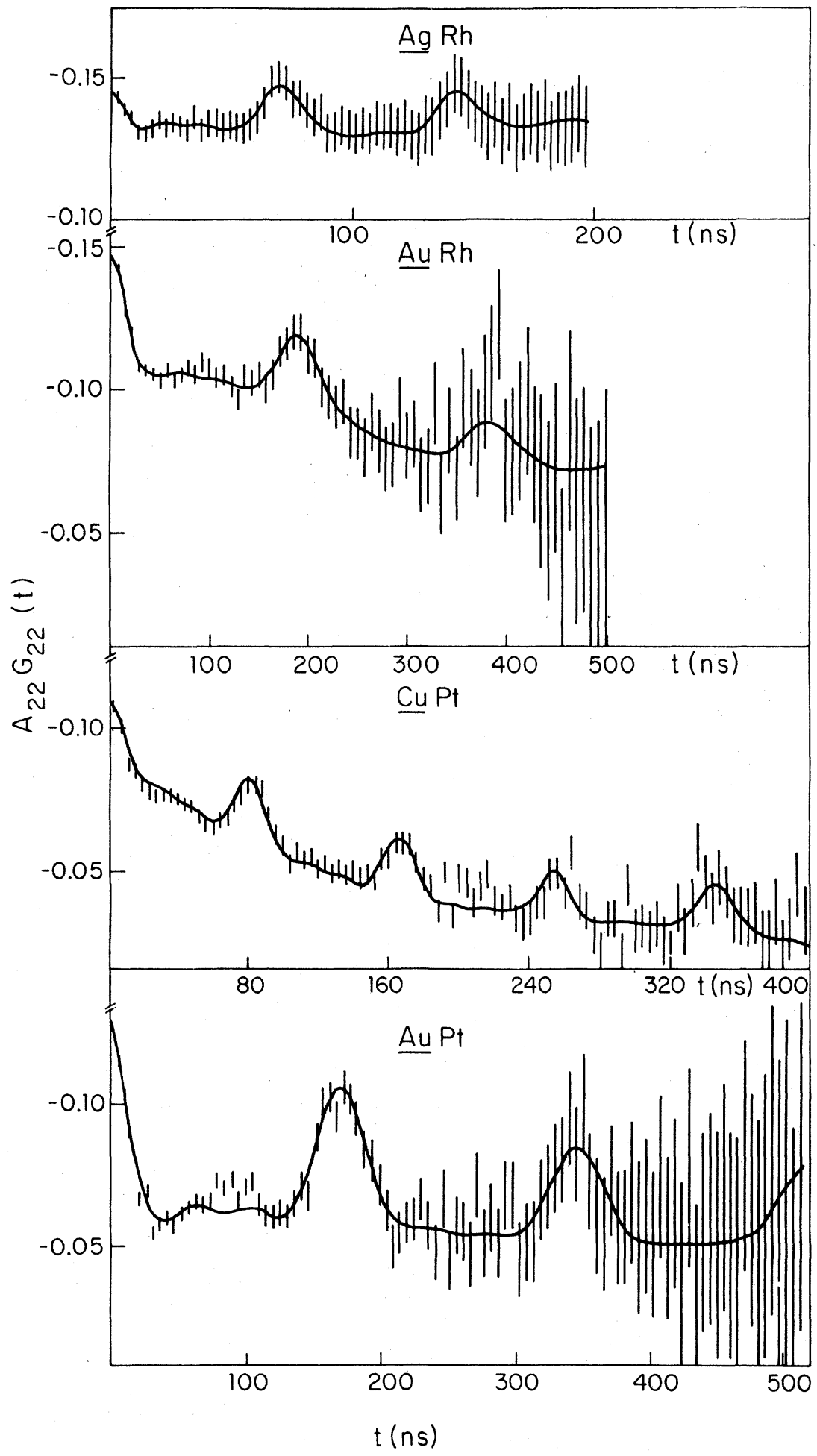

FIG. 1. Typical $A_{22} G_{22}(t)$ spectra for various alloys. The full lines are computer least-squares fittings. 


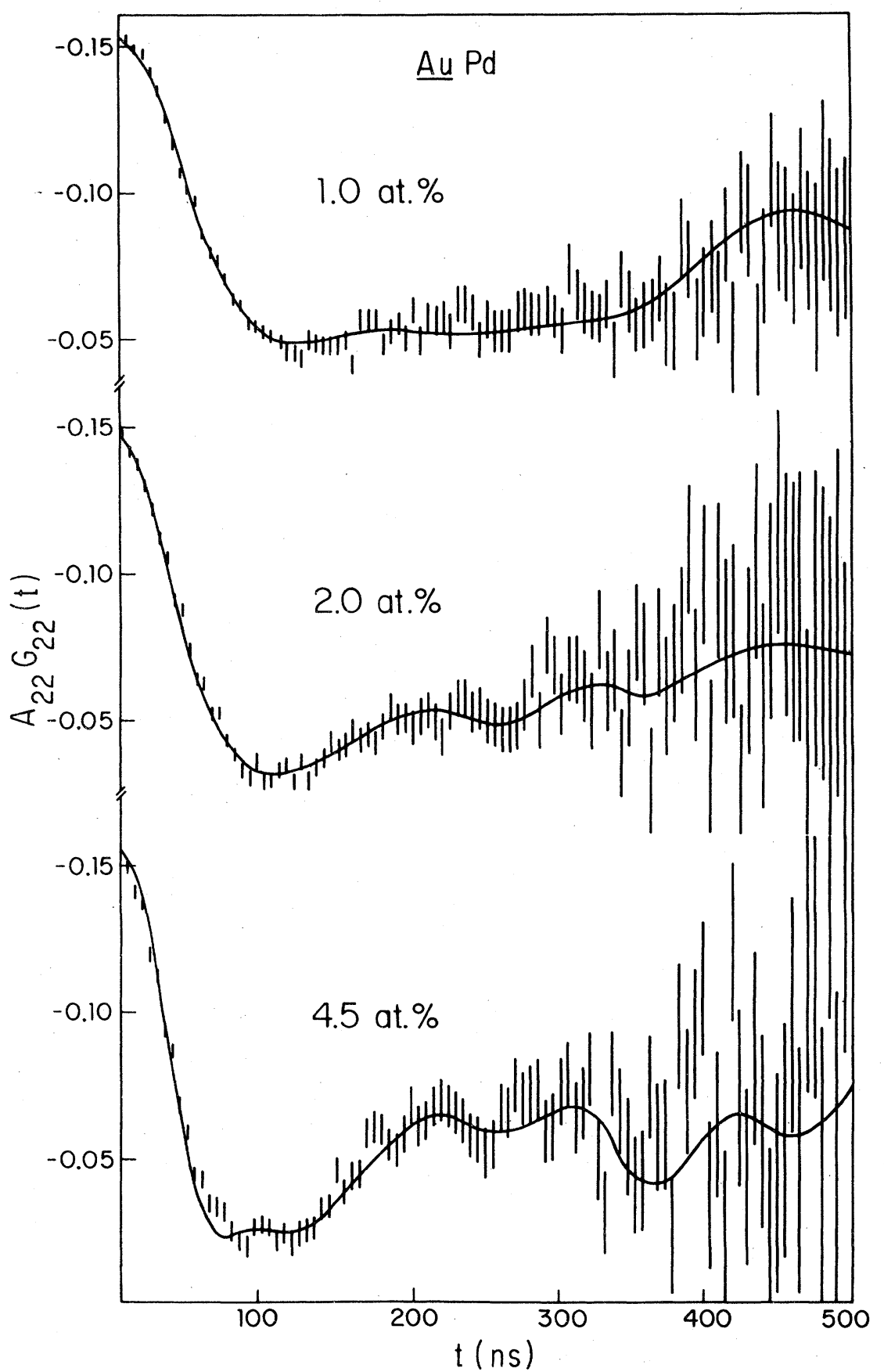

FIG. 2. $A_{22} G_{22}(t)$ curves for three concentrations of Pd in Au host. The full lines are computer least-squares fittings.

well as the results for $A g \mathrm{Pt}^{5,6}$

Figure 2 shows typical $A_{22} G_{22}(t)$ results at various impurity concentrations in $A u \mathrm{Pd}$. The extracted parameters are shown in Table III for impurity concentrations in the range $0.5-4.5$ at. \%.

Figure 3 shows typical $A_{22} G_{22}(t)$ results at various temperatures in $C u \mathrm{Rh}$ with 0.5 at. \% Rh. Table IV displays the results of the temperature-dependence study for the alloys of $A g \mathrm{Rh}(0.25$ at. \%), $C u \mathrm{Rh}(0.5$ at. \%), and $C u P t(1.0$ at. \%).

\section{A. Concentration dependence}

For two of the investigated alloys, $A u \mathrm{Pd}$ and $A g \mathrm{Rh}$, the concentration of impurity was changed. 


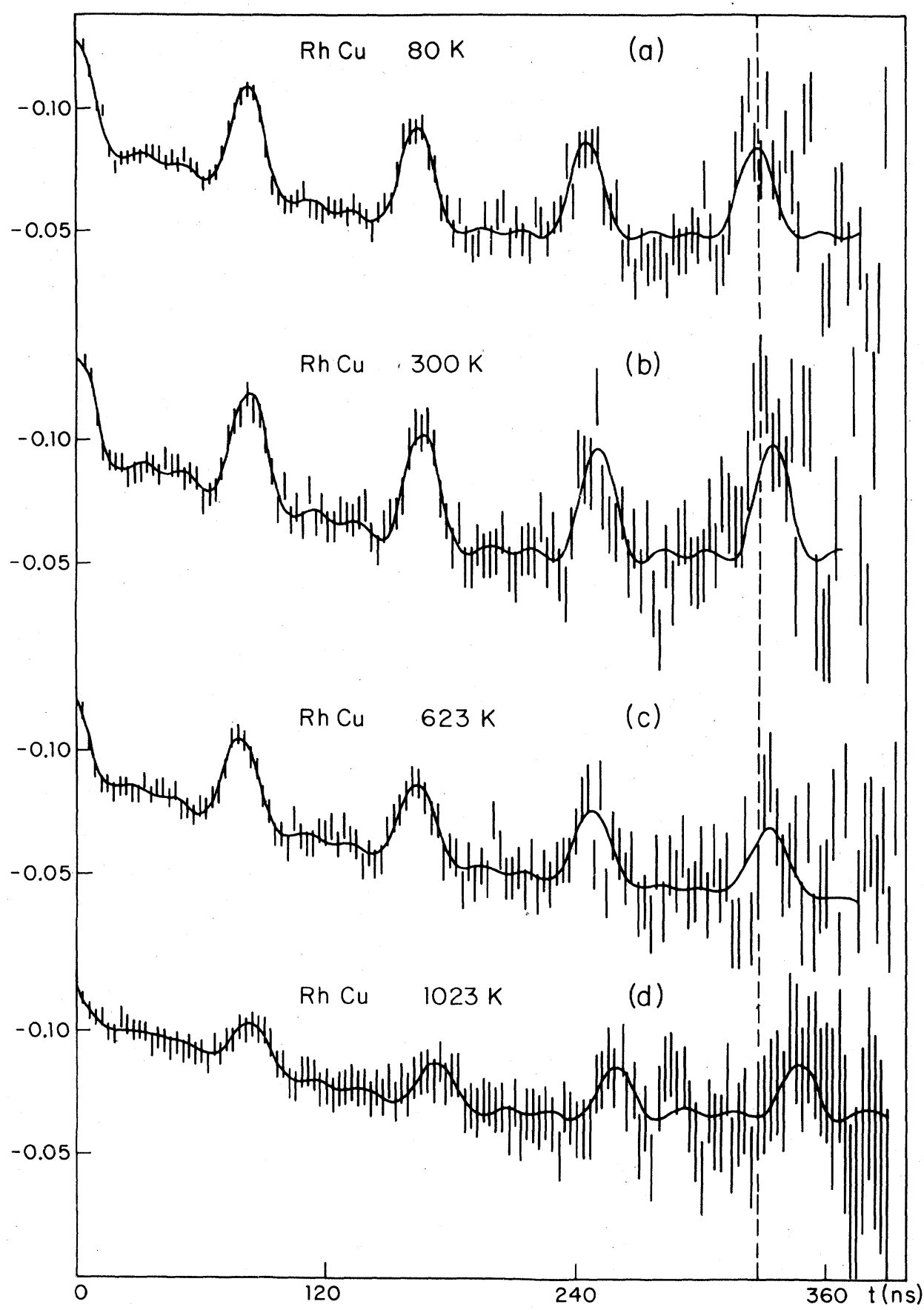

FIG. 3. $A_{22} G_{22}(t)$ curves for 0.5 at. \% of Rh impurity in $\mathrm{Cu}$, measured at different temperatures.

Since the available information in the literature ${ }^{8}$ indicates that the solubility of $\mathrm{Rh}$ in $\mathrm{Ag}$ is small, we measured the EFG only for concentrations of 0.25 and 0.5 at. \%. The computer fitting of the $A_{22} G_{22}(t)$ curves gave the same parameters for both concentrations. Figure 1 shows the result for 0.25 at. $\%$ of concentration and the extracted parameters are in Table II. The observation that the amplitudes and the frequencies are the same for both concentrations indicates that there is a segregation of the impurity. This fact is confirmed by the lack of any lowfrequency interaction (due to distant neighbors to the probe), in opposition to what is observed for all other impurities. Very likely the segregation starts at lower concentrations than 0.25 at. \%.

The $A u \mathrm{Pd}$ results are displayed in Table III. As 
TABLE II. Quadrupole interaction frequencies, with their respective amplitudes and distributions, for several alloys with the lowest concentration of impurity (see Table I). The errors on the amplitudes $a$ are typically $10 \%$.

\begin{tabular}{|c|c|c|c|c|c|c|c|c|}
\hline System & $\begin{array}{c}\text { Impurity } \\
\text { Concentration } \\
\text { (at. \%) }\end{array}$ & $a_{0}$ & $a_{l}$ & $\begin{array}{c}\nu_{l} \\
(\mathrm{MHz})\end{array}$ & $\delta_{l}$ & $a_{h}$ & $\begin{array}{c}v_{h} \\
(\mathrm{MHz})\end{array}$ & $\delta_{h}$ \\
\hline$C u \mathrm{Pd}^{\mathrm{a}}$ & 0.5 & 0.08 & 0.64 & $2.63 \pm 0.20$ & $0.50 \pm 0.10$ & 0.28 & $52.8 \pm 0.5$ & 0.01 \\
\hline - $C u P t$ & 1.0 & 0.23 & 0.56 & $6.2 \pm 0.6$ & $0.50 \pm 0.10$ & 0.21 & $80.2 \pm 0.8$ & 0.01 \\
\hline$C u \mathrm{Rh}$ & 0.5 & 0.27 & 0.37 & $6.6 \pm 0.5$ & $0.15 \pm 0.02$ & 0.36 & $80.2 \pm 0.8$ & 0.01 \\
\hline$A g \mathrm{Pd}^{\mathrm{b}}$ & 0.25 & 0.65 & 0.05 & $6.66 \pm 0.67$ & $0.40 \pm 0.04$ & 0.30 & $43.6 \pm 0.9$ & 0.01 \\
\hline$A g \mathrm{Rh}$ & 0.25 & 0.91 & $\cdots$ & $\ldots$ & $\ldots$ & 0.09 & $75.1 \pm 1.0$ & 0.01 \\
\hline$A g \operatorname{Ir}^{\mathrm{c}}$ & 0.2 & 0.51 & 0.46 & $2.9 \pm 0.4$ & $0.30 \pm 0.10$ & 0.03 & $201.0 \pm 6.0$ & 0.01 \\
\hline$A g \mathrm{Pt}^{\mathrm{d}}$ & 0.3 & $\approx 0.50$ & $\approx 0.15$ & 0 & $\cdots$ & $\approx 0.38$ & $76.5 \pm 0.8$ & $\cdots$ \\
\hline$A u \mathrm{Pd}$ & 0.5 & 0.22 & 0.14 & $0.80 \pm 0.10$ & $0.22 \pm 0.04$ & 0.64 & $14.1 \pm 1.0$ & $0.15 \pm 0.02$ \\
\hline$A u P t$ & 0.5 & 0.46 & 0.07 & $3.9 \pm 0.2$ & $0.29 \pm 0.04$ & 0.47 & $38.9 \pm 0.5$ & $0.04 \pm 0.01$ \\
\hline$A u \mathrm{Rh}$ & 0.5 & 0.50 & 0.22 & $3.6 \pm 0.6$ & $0.10 \pm 0.02$ & 0.28 & $35.7 \pm 0.5$ & $0.06 \pm 0.02$ \\
\hline
\end{tabular}

a From Ref. 4.

${ }^{b}$ From Ref. 3.

\footnotetext{
'Preliminary results.

${ }^{\mathrm{d} A m p l i t u d e s}$ estimated from Ref. 6.
}

with the previously reported experiments for $A g \mathrm{Pd}$ and $C u P d$, we see the onset of new high frequencies as the impurity concentration is increased. At 0.5 at. $\%$ two frequencies are present, one very low, $\nu_{l}=0.8 \mathrm{MHz}$, with $22 \%$ of distribution and a second one, $v_{h}{ }^{1}, 20$ times larger with a similarly large distribution of $15 \%$. When the concentration is increased, the low frequency disappears, the distribution of $\nu_{h}^{1}$ decreases to $1 \%$ and two new sharp frequencies $\nu_{h}^{2}$ and $\nu_{h}^{3}$, larger than $v_{h}^{1}$ appear. In accordance with the interpretation given in Ref. 3, the frequency $\nu_{h}^{1}$ is due to one NN impurity atom, the frequencies $\nu_{h}^{2}$ and $\nu_{h}^{3}$ are generated by different configurations of two NN impurities and the smeared-out low-frequency $\nu_{l}$ comes from impurities located at larger than NN distances from the probe nucleus. The amplitudes of the high frequencies are strongly enhanced over a random distribution of impurities, showing again the presence of a strong attraction between the In probe and the Pd-impurity atoms. For 0.5 at. \% of impurity the enhancement factor $f_{h}$ of the amplitude for the high frequency is of the order of 10 .

\section{B. Temperature dependence}

The effect of temperature on the EFG was measured for the three alloys, $C u \mathrm{Rh}(0.5$ at. \%), $C u \mathrm{Pt}$ (1.0 at. \%), and $A g \mathrm{Rh}(0.25$ at. \%). The results are shown in Table IV. For each alloy the highfrequency quadrupole interaction follows the empirical $T^{3 / 2}$ temperature dependence ${ }^{10}$ :

$$
\nu(T)=\nu(0)\left(1-\alpha T^{3 / 2}\right) .
$$

TABLE III. Parameters extracted from the fitting of $A_{22} G_{22}(t)$ curves for different concentrations of Pd impurity in Au matrix at room temperature. (The fitting for $\nu_{l}$ and $\nu_{h}^{1}$ gave $\eta=0$. Typical errors are $10 \%$ for $\nu_{l}, \delta$, and $\eta$, $7 \%$ for $\nu_{h}$, and $10 \%$ for $a$.)

\begin{tabular}{|c|c|c|c|c|c|c|c|c|c|c|c|c|c|c|c|}
\hline $\begin{array}{c}C \\
(\text { at. } \%)\end{array}$ & $a_{0}$ & $a_{l}$ & $\begin{array}{c}\nu_{l} \\
(\mathrm{MHz})\end{array}$ & $\delta_{l}$ & $a_{h}^{1}$ & $\begin{array}{c}\nu_{h}^{1} \\
(\mathrm{MHz})\end{array}$ & $\delta_{h}^{1}$ & $a_{h}^{2}$ & $\begin{array}{c}v_{h}^{2} \\
(\mathrm{MHz})\end{array}$ & $\delta_{h}^{2}$ & $\eta_{h}^{2}$ & $a_{h}^{3}$ & $\begin{array}{c}v_{h}^{3} \\
(\mathrm{MHz})\end{array}$ & $\delta_{h}^{3}$ & $\eta_{h}^{3}$ \\
\hline 0.5 & 0.22 & 0.14 & 0.8 & 0.22 & 0.64 & 14.1 & 0.15 & $\ldots$ & $\cdots$ & $\ldots$ & $\cdots$ & $\cdots$ & $\ldots$ & $\ldots$ & $\cdots$ \\
\hline 1.0 & 0.30 & $\ldots$ & $\ldots$ & $\ldots$ & 0.70 & 14.3 & 0.13 & $\ldots$ & $\ldots$ & $\ldots$ & $\ldots$ & $\ldots$ & & $\ldots$ & . \\
\hline 2.0 & 0.25 & $\ldots$ & $\ldots$ & $\cdots$ & 0.45 & 14.8 & 0.06 & 0.30 & 19.8 & 0.05 & 0.45 & $\ldots$ & $\ldots$ & $\ldots$ & $\ldots$ \\
\hline 4.5 & 0.20 & $\ldots$ & $\ldots$ & $\cdots$ & 0.20 & 15.4 & 0.01 & 0.38 & 20.2 & 0.05 & 0.45 & 0.22 & 26.3 & 0.05 & 0.40 \\
\hline
\end{tabular}


TABLE IV. Parameters extracted from the temperature dependence of the EFG for the $C u \mathrm{Rh}, C u \mathrm{Pt}$, and $A g \mathrm{Rh}$ systems. (The typical errors are $10 \%$ for $a_{0}, a_{l}$, and $a_{h}, 20 \%$ for $\nu_{l}$ and $\delta_{l}$, and $1 \%$ for $\nu_{h}$.)

\begin{tabular}{|c|c|c|c|c|c|c|c|}
\hline & $\begin{array}{c}T \\
(\mathrm{~K})\end{array}$ & $a_{0}$ & $a_{l}$ & $\begin{array}{c}\nu_{l} \\
(\mathrm{MHz})\end{array}$ & $\delta_{l}$ & $a_{h}$ & $\begin{array}{c}\nu_{h} \\
(\mathrm{MHz})\end{array}$ \\
\hline & 80 & 0.33 & 0.32 & 6.8 & 0.3 & 0.35 & 81.9 \\
\hline & 300 & 0.27 & 0.37 & 6.6 & 0.15 & 0.36 & 80.2 \\
\hline & 523 & 0.15 & 0.53 & 3.7 & 0.61 & 0.32 & 79.2 \\
\hline & 573 & 0.18 & 0.54 & 3.0 & 0.95 & 0.28 & 79.0 \\
\hline \multicolumn{8}{|l|}{$C u R h$} \\
\hline & 623 & 0.18 & 0.57 & 3.0 & $\begin{array}{l}0.97 \\
0.8\end{array}$ & 0.25 & $\begin{array}{l}79.5 \\
768\end{array}$ \\
\hline & 723 & 0.18 & 0.60 & $\begin{array}{l}3.2 \\
50\end{array}$ & $\begin{array}{l}0.8 \\
0.5\end{array}$ & 0.22 & 76.8 \\
\hline & 873 & 0.38 & 0.44 & 5.9 & 0.5 & 0.18 & 75.7 \\
\hline & 1023 & 0.55 & 0.30 & 6.7 & 0.27 & 0.15 & 75.1 \\
\hline & 80 & 0.22 & 0.57 & 6.01 & 0.5 & 0.21 & 81.5 \\
\hline & 300 & 0.23 & 0.56 & 6.2 & 0.5 & 0.21 & 80.2 \\
\hline & 573 & 0.41 & 0.38 & 9.79 & 0.21 & 0.21 & 79.0 \\
\hline & 673 & 0.36 & 0.46 & 8.67 & 0.25 & 0.19 & 79.8 \\
\hline \multicolumn{8}{|l|}{$C u \mathrm{Pt}$} \\
\hline & 773 & 0.32 & 0.51 & 7.86 & 0.38 & 0.17 & 77.6 \\
\hline & 873 & 0.20 & 0.64 & 5.80 & 0.62 & 0.16 & 76.2 \\
\hline & 973 & 0.26 & 0.59 & 4.98 & 0.70 & 0.15 & 75.7 \\
\hline & 1023 & 0.16 & 0.71 & 4.15 & 0.60 & 0.13 & 75.0 \\
\hline & 80 & 0.91 & & & & 0.09 & 76.0 \\
\hline & 300 & 0.91 & & & & 0.09 & 75.1 \\
\hline \multicolumn{8}{|l|}{$A g \mathrm{Rh}$} \\
\hline & 523 & 0.90 & & & & 0.10 & 73.8 \\
\hline & 673 & 0.90 & & & & 0.10 & 73.2 \\
\hline
\end{tabular}

TABLE V. Enhancement factors $f_{h}$ and $F_{h}$ and parameters $\alpha, \Delta E_{B}$, and $T_{0}$ obtained from temperature experiments for various alloys.

\begin{tabular}{|c|c|c|c|c|c|c|c|c|c|}
\hline Alloy & $\begin{array}{c}\text { Impurity } \\
\text { concentration } \\
(\text { at. } \%)\end{array}$ & $f_{h}$ & $\stackrel{\alpha}{\alpha}\left(10^{-5} \mathrm{~K}^{-3 / 2}\right)$ & $\begin{array}{c}\Delta E_{B} \\
(\mathrm{meV})\end{array}$ & $\begin{array}{l}T_{0} \\
(\mathrm{~K})\end{array}$ & $F_{h}$ & $\ln F_{h}$ & $\frac{\Delta E_{B}}{k T_{0}}$ & $\begin{array}{c}\alpha \Delta E_{B} \\
\left(10^{-8} \mathrm{eV} \mathrm{K}^{-3 / 2}\right)\end{array}$ \\
\hline$C u \mathrm{Pt}_{\mathrm{t}}$ & 1.0 & 1.8 & $0.25 \pm 0.02$ & $60 \pm 15$ & 620 & 2.2 & 0.79 & 1.12 & $15 \pm 4$ \\
\hline$C u \mathrm{Pd}^{\mathrm{b}}$ & 0.5 & 4.7 & $0.31 \pm 0.05$ & $100 \pm 40$ & 600 & 6.4 & 1.9 & 1.9 & $31 \pm 14$ \\
\hline$C u R h$ & 0.5 & 6.0 & $0.29 \pm 0.02$ & $86 \pm 8$ & 430 & 9.3 & 2.2 & 2.3 & $25 \pm 3$ \\
\hline$A g \mathbf{P d}^{\mathrm{c}}$ & 0.25 & 10 & $0.18 \pm 0.04$ & $122 \pm 20$ & 710 & 14 & 2.7 & 2.0 & $22 \pm 6$ \\
\hline$A g \mathrm{Pd}^{\mathrm{d}}$ & 0.3 & $\approx 9$ & $0.203 \pm 0.025$ & $135 \pm 9$ & $\approx 570$ & $\approx 13$ & $\approx 2.6$ & $\approx 2.7$ & $27 \pm 4$ \\
\hline$A g \mathrm{Pt}^{\mathrm{d}}$ & 0.3 & $\approx 10$ & $0.176 \pm 0.011$ & $171 \pm 9$ & $\approx 600$ & $\approx 17$ & $\approx 2.8$ & $\approx 3.3$ & $30 \pm 3$ \\
\hline
\end{tabular}

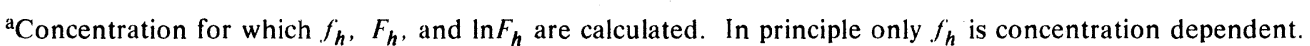

${ }^{b}$ Reference 4.

'Reference 3 .

${ }^{\mathrm{d}}$ References 5 and 6 . The values of $T_{0}$ are from the crossing points in Fig. 2 of Ref. 6. 
The extracted $\alpha$ coefficients from the fittings are shown in Table V, which also includes results from previous measurements.

The $C u \mathrm{Rh}$ and $C u \mathrm{Pt}$ systems were studied in the temperature range from 80 to $1023 \mathrm{~K}$. As well as the change of the quadrupole frequency, Table IV also shows that, for these two alloys, the amplitude $a_{h}$ of the high frequency decreases at high temperatures. Simultaneously the sum of the amplitude $a_{0}+a_{l}$ (background plus low-frequency amplitudes) increases in the same temperature range. We associate the high frequency with bound In-impurity pairs ( $\operatorname{In} X)$, the impurity being $\mathrm{NN}$ to the probe In atom, and these have a fractional population $a_{h}$. For the remainder of the population $\left(a_{0}+a_{l}\right)$, the In probe atoms have the impurity beyond the NN, feel a lowfrequency interaction or none at all, and are represented by $(\operatorname{In}+X)$. Our data show that, as the temperature increases we have the chemical reactions $(\operatorname{In} X) \rightleftharpoons(\mathrm{In}+X)$, with the net result that at $1023 \mathrm{~K}$ about $50 \%$ of the $(\operatorname{In} X)$ bound pairs present at $80 \mathrm{~K}$ were broken and transformed into distant neighbors. The measured amplitude variation with temperature was found to be independent of the order of the different temperature runs using an average rate of change of temperature between runs of about $10^{-2} \mathrm{~K} \mathrm{~s}^{-1}$, and run times of the order of $12 \mathrm{~h}$.

The data of Table IV allow the determination of the difference in binding energy $\Delta E_{B}$, between the systems $(\operatorname{In} X)$ and $(\operatorname{In}+X)$, for $X=\mathrm{Rh}, \mathrm{Pt}$ in a $\mathrm{Cu}$ host. Assuming the validity of the law of mass action and neglecting entropy effects, we can write

$$
\ln \frac{a(\operatorname{In} X)}{a(\operatorname{In}+X)}=\ln \frac{a_{h}}{a_{0}+a_{l}}=\frac{\Delta E_{B}}{k T} .
$$

Figure 4 shows the experimental values of $a_{h} /\left(a_{0}+a_{l}\right)$ plotted on a logarithmic scale against $T^{-1}$. In the high-temperature region the data are fit- ted very well by a straight line. Up to about $430 \mathrm{~K}$ in $C u \mathrm{Rh}$ and about $620 \mathrm{~K}$ in $C u \mathrm{Pt}$, the impurity and probe atoms are not mobile and for these temperatures Eq. (5) is not obeyed. The results obtained for the difference in binding energy $\Delta E_{B}$ are $86 \pm 8 \mathrm{meV}$ in $C u \mathrm{Rh}$ and $60 \pm 15 \mathrm{meV}$ in $C u \mathrm{Pt}$. These are compared in Table $\mathrm{V}$ with previous measurements in other doped alloys.

For the $A g \mathrm{Rh}$ alloy, the EFG also follows the $T^{3 / 2}$ law but the amplitudes are constant at least up to 673 $\mathrm{K}$, the highest measured temperature. With the present data we cannot discriminate between a normal detrapping process for $A g \mathrm{Rh}$, but beginning above $673 \mathrm{~K}$, or a process where detrapping is compensated by some means.

\section{DISCUSSION OF SYSTEMATIC TRENDS}

\section{A. Impurity-probe interaction}

Table II shows the frequencies, amplitudes, and distributions obtained at lowest measured impurity concentration for the various alloys studied in this paper and in previous work. All the impurities are to the left of the noble-metal hosts in the Periodic Table and in each case there is only one high frequency $\nu_{h}$ which is associated with one impurity atom NN to the probe. For nine of the ten alloys listed, the exception being $A g \mathrm{Ir}$, there is a strong enhancement of NN populations over that expected from a random distribution of impurities. This signifies an attraction between the In probe and the impurity atoms. The enhancement factor $f_{h}\left(=a_{h} / 12 c^{\prime}\right.$ where $c^{\prime}$ is the fractional impurity concentration) ranges from 2 in $C u$ Pt to about 10 in $A g \mathrm{Pd}, A g \mathrm{Pt}$, and $A u \mathrm{Pd}$. We shall also see that the apparent exception $A g$ Ir fits into a scheme of varying enhancement for the different impurity and host combinations.

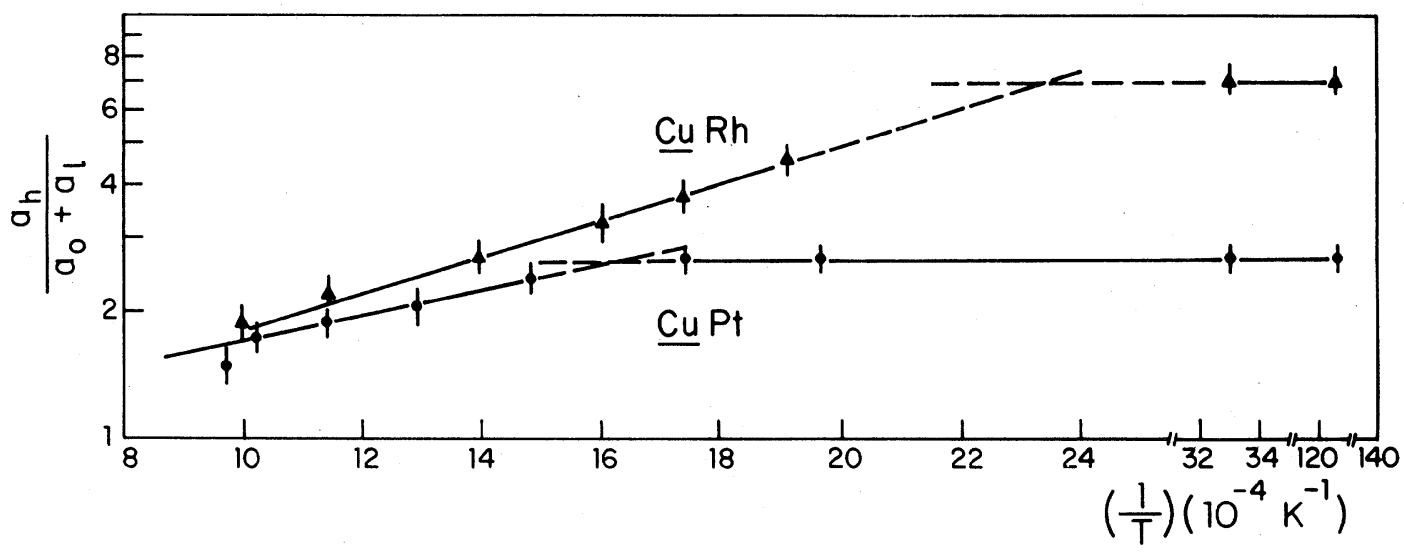

FIG. 4. Experimental values of $a_{h} /\left(a_{0}+a_{l}\right)$ on a logarithmic scale vs $1 / T$ for $C u$ Rh and $C u$ Pt. The lines show the linear fittings (see text). 
For those alloys where temperature effects have been measured, there is, as might be expected, a rough correlation between the enhancement factor $f_{h}$ and the binding energy $\Delta E_{B}$. Table $\mathrm{V}$ shows that high $f_{h}$ is associated with high $\Delta E_{B}$. A more precise relationship between these two quantities can be obtained after including the neglected entropy effects in Eq. (5). If the system is in thermodynamic equilibrium

$$
\ln \left(\frac{a_{h}}{a_{0}+a_{l}}\right)=\frac{\Delta E_{B}}{k T}+\frac{1}{k} \Delta S,
$$

where $\Delta S$ is the difference in configurational entropy between the state with one impurity NN to the probe and the state with no impurity NN to the probe.

Our enhancement factors apply at low temperatures where the alloys are not in thermodynamic equilibrium. Instead, constant values of the amplitudes were obtained. If we define $T_{0}$ as the temperature of intersection of the low- and high-temperature lines in Fig. 4 then the low-temperature amplitudes obey

$$
\ln \left(\frac{a_{h}}{a_{0}+a_{l}}\right)=\frac{\Delta E_{B}}{k T_{0}}+\frac{1}{k} \Delta S .
$$

$T_{0}$ indicates the temperature region where transformation between the high-temperature equilibrium situation and the low-temperature frozen situation occurs. It could in principle be related to the thermal treatment of the sample and impurity diffusion rates.

If no more than one impurity $\mathrm{NN}$ to the probe is observed $\left(a_{0}+a_{l}+a_{h}=1\right)$, the left-hand side of Eq. (7) becomes $\ln \left[a_{h} /\left(1-a_{h}\right)\right]$ and a relationship between the enhancement factor and the binding energy immediately results. Where configurations with more than one impurity $\mathrm{NN}$ to the probe occur, the one NN impurity amplitude $\left(a_{h}\right)$ can conveniently be renormalized to $a_{h}^{\prime}=a_{h} /\left(a_{0}+a_{l}+a_{h}\right)$, giving the left-hand side of Eq. (7) as $\ln \left[a_{h}^{\prime} /\left(1-a_{h}^{\prime}\right)\right]$. The simplest approximation for evaluating the entropy term in Eq. (7) is to allow all possible arrangements of one In probe atom and $c^{\prime} N$ impurity atoms on the $N$ lattice sites of the crystal. Then, neglecting surface effects,

$$
\begin{aligned}
\frac{1}{k} \Delta S & =\ln \left(\frac{12 N(N-13) ! /\left(c^{\prime} N-1\right) !\left(N-c^{\prime} N-12\right) !}{N(N-13) ! /\left(c^{\prime} N\right) !\left(N-c^{\prime} N-13\right) !}\right) \\
& =\ln \left[12 c^{\prime} /\left(1-c^{\prime}\right)\right]
\end{aligned}
$$

for large $N$.

Thus, the modified enhancement factor $F_{h}$, defined by

$$
F_{h}=\frac{a_{h}^{\prime} /\left(1-a_{h}^{\prime}\right)}{12 c^{\prime} /\left(1-c^{\prime}\right)},
$$

is related to the binding energy by

$$
\ln F_{h}=\Delta E_{B} / k T_{0} \text {. }
$$

$F_{h}$ is the ratio of observed to randomly distributed populations for one impurity NN to the probe, each being expressed relative to the populations for no impurity NN to the probe.

The experimental results have been analyzed on this basis in Table $V$. There is a very good comparison between $\ln F_{h}$ and $\Delta E_{B} / k T_{0}$, much better than the rough correlation between $f_{h}$ and $\Delta E_{B}$. The data from Refs. 3 and 6 for $A g$ Pd are treated separately because of differences in sample treatments. The less satisfactory comparison for the Ref. 3 results is most probably due to the quenching used being too slow, resulting in an unnaturally high $T_{0}$.

The plausibility of the obtained values of $T_{0}$ can be checked using known impurity diffusion constants and the sample treatment. In our experiments the samples spent typically $3 \mathrm{~h}$ at measurement temperature before and in the early part of the temperature runs. Allowing several jumps for either the probe In or the transition-metal impurity to occur in this time we estimate that a minimum jump rate of $10^{-3} \mathrm{~s}^{-1}$ is required to establish equilibrium for the bulk of the measurement period. The only case where both In and impurity diffusion constants in the host metal are known is $A g \mathrm{Pd}$ and here In is the most mobile giving a temperature of $510 \mathrm{~K}$ for the above jump rate. This is in reasonable agreement with the preferred value for $T_{0}$ of $570 \mathrm{~K}$, although, since this is obtained from Ref. 6 , the required jump rate is uncertain. Fortunately the calculated temperature depends only weakly on the minimum jump rate, a change by a factor of 10 in the jump rate producing only a $5 \%$ change in the temperature. In addition, the above treatment must be viewed as an approximation since it assumes that the measured tracer diffusion rates are valid in the presence of the impurity-probe interaction.

With the In diffusion identified as responsible for the value of $T_{0}$ in $A g \mathrm{Pd}$, the value of $T_{0}$ in $\mathrm{AgPt}$ is expected to be the same or lower. Although the values in Table $\mathrm{V}$ show the reverse of this the difference is small. Also the low value of $T_{0}$ in $C u \mathrm{Rh}$ compared to the other two $\mathrm{Cu}$ alloys indicates that there the diffusion of $\mathrm{Rh}$ is the important factor.

The obvious quantity to use in a systematic study of the probe-impurity interaction in the various alloys is the binding energy $\Delta E_{B}$. Since it has been determined for only one-half of the studied alloys, it is estimated for the others using the measured lowtemperature amplitudes and Eq. (10). This involves an estimation of the temperature $T_{0}$ which is set at $600 \mathrm{~K}$ from the indications of Table $\mathrm{V}$, unless there is evidence to the contrary. This value probably has an uncertainty of at most $30 \%$. For $A g \mathrm{Rh}$ the amplitude $a_{h}$ was found to be constant up to $673 \mathrm{~K}$ and we have set $T_{0}$ for this alloy at $700 \mathrm{~K}$.

Trends between measured binding energies and EFG effects are discussed in Sec. IV B and trends 
among binding energies, including estimated values, are analyzed in Sec. IV C.

\section{B. Electric-field-gradient behavior}

The room-temperature values of the highfrequency $\nu_{h}$ for all the alloys are analyzed in Sec. IV C on the basis of host and impurity position in the Periodic Table.

For alloys where temperature effects have been measured the high-frequency $\nu_{h}$ follows the empirical $T^{3 / 2}$ temperature dependence [Eq. (4)]. This strongly suggests that the cause of the EFG temperature dependence in dilute cubic alloys is the same as in noncubic metals, arising from the thermal vibrations of the atoms. ${ }^{11-13}$ This idea is further strengthened by an apparent correlation between the binding energy and the $\alpha$ parameter which gives the strength of the temperature dependence. If the temperature dependence of the EFG is governed by thermal vibrations, for strongly bound systems (higher binding energy) one would expect a smaller temperature dependence of the EFG. This fact is confirmed experimentally; the values of the $\alpha$ parameter are smaller for strongly bound probe-impurity pairs and larger for weakly bound pairs. This correlation can be observed more clearly from the product $\alpha \Delta E_{B}$ listed in Table $\mathrm{V}$ which, with the possible exception of the $C u \mathrm{Pt}$ alloy, is approximately constant while $\Delta E_{B}$ varies by a factor of about 2 .

It should be emphasized that the probe-impurity interaction of importance to the value of $\alpha$ applies to the situation when the probe is ${ }^{111} \mathrm{Cd}$, whereas the measured binding energies apply to the situation when the impurity population is in thermodynamic equilibrium and the probe is still ${ }^{111} \mathrm{In}$. Therefore, in making the preceding argument connecting $\alpha$ and $\Delta E_{B}$ we must assume that the measured In-impurity binding energies give a measure of the Cd-impurity binding energies. Furthermore, within the vibratingatom picture of the temperature dependence, one really expects that $\alpha$ should depend in some manner on the hardness of the potentials seen by the vibrating atoms contributing to the observed EFG and also on the masses of these atoms. ${ }^{14}$ Though it is natural to expect the hardness of a potential to be related to its depth, a description of $\alpha$ just in terms of the binding energy of the one NN impurity configuration can be expected to give only qualitative agreement at best. An improvement would be to use $\alpha$ values with the mass effects eliminated, but current theory ${ }^{15}$ is only applicable to the Ag alloys where the probe and host atoms are of similar mass. It is interesting that this theory predicts a decrease in $\alpha$ for large impurity to host mass ratio, in line with the exceptionally small $\alpha \Delta E_{B}$ in $C u \mathrm{Pt}$ where the mass ratio is largest.
A further trend involving $\alpha$ values and binding can be seen on comparing the temperature dependence of the high and low frequencies, due, respectively, to $\mathrm{NN}$ and distant neighbors. For the high frequencies, associated with strongly bound probe-impurity pairs, $\alpha$ is in the range $0.2-0.3 \times 10^{-5} \mathrm{~K}^{-3 / 2}$ (Table V). The low-frequency distributions have a much stronger temperature dependence, with $\alpha$ an order of magnitude larger. In the $A g \mathrm{Pd}$ alloy ${ }^{3}$ the low smeared-out frequency had $\alpha$ as $(3.1 \pm 0.6) \times 10^{-5} \mathrm{~K}^{-3 / 2}$. From the data of Table IV similar values can be obtained for $C u \mathrm{Rh}$ in the range $80-700 \mathrm{~K}$ and for $C u \mathrm{Pt}$ in the range 600 to $1000 \mathrm{~K}$. This failure of the lowfrequency distribution to hold to the $T^{3 / 2}$ dependence throughout the complete temperature range may be due to thermal changes in the distribution of impurities between different non-NN shells.

We do not suggest that the reciprocal relation between $\alpha$ and binding energy, evidenced for the NN impurity interaction, also holds for comparing NN and non-NN impurity interactions. Obviously for a very distant impurity, where impurity-probe binding is negligible, the associated EFG would be expected to have a temperature dependence governed by the independent vibration of the probe and the impurity relative to the host. However the trend of decreasing $\alpha$ with increasing impurity-probe binding is apparent and supports the interpretation of the EFG temperature dependence as arising from the thermal vibrations of the atoms.

In connection with the above discussion it is interesting to note that for the $\mathrm{Ag} \mathrm{Pd}_{\text {alloy }}{ }^{3}$ the high frequency generated by one $\mathrm{NN}$ impurity of $\mathrm{Pd}$ had a weaker temperature dependence $[\alpha=(0.18 \pm 0.04)$ $\left.\times 10^{-5} \mathrm{~K}^{-3 / 2}\right]$ than the frequency due to two $\mathrm{NN}$ of $\operatorname{Pd}\left[\alpha=(0.44 \pm 0.10) \times 10^{-5} K^{-3 / 2}\right]$. It seems that in this alloy each impurity in the two NN configuration is bound more weakly (to the probe atom) than in the configuration of one $\mathrm{NN}$, perhaps due to the repulsion between impurities.

\section{Periodic Table analysis}

In the following we analyze the high-frequency data of Table II and the binding-energy results of Table $\mathbf{V}$ in terms of the valence difference between impurity and host, size effects and quantum numbers of the last $d$ shell of hosts and impurities. Since the valence of a transition-metal ion in a metallic environment is not obvious we take the valence as that used in the transition-metal model potential of Animalu. ${ }^{16}$ Such potentials are applicable in principle to a theoretical treatment of the systems under study.

We thus have the following assumptions: (i) There is an effective difference of valence between impurity and host $[\Delta Z=Z$ (impurity) $-Z$ (host) $]$ of 
TABLE VI. High frequencies $\nu_{h}$ and binding energies $\Delta E_{B}$ for the measured alloys arranged as function of valence difference and atomic period.

\begin{tabular}{|c|c|c|c|c|c|c|c|c|c|c|c|c|}
\hline & Impurity & Host & $\lambda$ & Alloy & $\begin{array}{c}\Delta Z=1 \\
v_{h} \\
(\mathrm{MHz})\end{array}$ & $\begin{array}{c}\Delta E_{B} \\
(\mathrm{meV})\end{array}$ & Alloy & $\begin{array}{c}\Delta Z=2 \\
v_{h} \\
(\mathrm{MHz})\end{array}$ & $\begin{array}{l}\Delta E_{B} \\
(\mathrm{meV})\end{array}$ & Alloy & $\begin{array}{c}\Delta Z=3 \\
v_{h} \\
(\mathrm{MHz})\end{array}$ & $\begin{array}{c}\Delta E_{B} \\
(\mathrm{meV})\end{array}$ \\
\hline \multirow{4}{*}{$\begin{array}{l}\mathrm{Ag}-\text { and } \mathrm{Au}- \\
\text { based alloys }\end{array}$} & $4 d$ & $5 d$ & -1 & $A u \mathrm{Pd}$ & 14 & $175^{\mathrm{a}}$ & $A u \mathrm{Rh}$ & 36 & $96^{a}$ & \multirow{6}{*}{$A g \mathrm{Ir}$} & \multirow{6}{*}{201} & \multirow{6}{*}{$13^{\mathrm{a}}$} \\
\hline & $4 d$ & $4 d$ & 0 & $A g \mathrm{Pd}$ & 44 & $135 \pm 9$ & $A g \mathrm{Rh}$ & 75 & $72^{\mathrm{a}}$ & & & \\
\hline & $5 d$ & $5 d$ & 0 & $A u \mathrm{Pt}$ & 39 & $139^{\mathrm{a}}$ & & & & & & \\
\hline & $5 d$ & $4 d$ & 1 & $A g \mathrm{Pt}$ & 76 & $171 \pm 9$ & & & & & & \\
\hline \multirow{2}{*}{$\begin{array}{c}\text { Cu-based } \\
\text { alloys }\end{array}$} & $4 d$ & $3 d$ & 1 & $C u \mathrm{Pd}$ & 53 & $100 \pm 40$ & \multirow[t]{2}{*}{$C u \mathrm{Rh}$} & \multirow[t]{2}{*}{80} & \multirow[t]{2}{*}{$86 \pm 8$} & & & \\
\hline & $5 d$ & $3 d$ & 2 & $C u \mathrm{Pt}$ & 80 & $60 \pm 15$ & & & & & & \\
\hline
\end{tabular}

$\overline{{ }^{a} \text { Estimated using Eq. (10) (see text) and probably in error by at most 30\%. Some uncertainty exists for the effective concentra- }}$ tion ( $\leqslant 0.25$ at. \%) in the $A g \mathrm{Rh}$ alloy.

$\Delta Z=1$ for $\mathrm{Pd}$ and $\mathrm{Pt}, \Delta Z=2$ for $\mathrm{Rh}$, and $\Delta Z=3$ for Ir. The value of $Z=4$ for Ir is justified in Ref. 16 . (ii) $\mathrm{We}$ define a parameter $\lambda$ as the difference between the principal quantum numbers of the last $d$ shell of the impurity and host atoms. In this way $\lambda$ will parametrize the effects of period in the Periodic Table. (iii) Because $\mathrm{Ag}$ and $\mathrm{Au}$ have similar atomic volumes and electronic band structures we shall compare and correlate the results for both hosts. The host of $\mathrm{Cu}$ has a different atomic volume and band structure; the $\mathrm{Cu}$-based alloys will be analyzed separately.

The high-frequency interaction $\nu_{h}$ and the corresponding binding energy $\Delta E_{B}$ are presented in Table VI for the various alloys and classified according to the above considerations. Also included are estimates of the binding energy as described in Sec. IV A for those alloys where $\Delta E_{B}$ was not measured.

The following facts emerge from an analysis of Table VI. (a) Independent of impurity and host, there is an increase of the frequency $\nu_{h}$ and an apparent decrease of the binding energy $\Delta E_{B}$ with increasing $\Delta Z$. (b) There is a correlation between the frequencies and the period parameter $\lambda$. The data for $\Delta Z=1$ show clearly that when $\lambda$ increases the frequency also increases. In addition, with the exception of $A g \mathrm{Pt}$, a decrease in $\Delta E_{B}$ with increasing $\lambda$ is apparent. (c) The frequencies for the impurities of $\mathrm{Pd}, \mathrm{Rh}$, and $\mathrm{Pt}$ in $\mathrm{Cu}$ host are systematically higher than in the other two hosts. This increase can be attributed, at least in part, to the fact that in the $\mathrm{Cu}$ host the distance between impurity and probe atoms is smaller than in the other two hosts.

From the correlation between $\Delta E_{B}$ and $\Delta Z$ we see that the lack of enhancement in $A g I r$ is not really an exceptional case, but fits naturally into a scheme of decreasing binding energy with increasing valence difference between impurity and host.

The increase of the high frequency with a positive nominal charge difference $\Delta Z$ was observed previously in TDPAC work with a $\mathrm{Ag}$ host $^{1}$ and also in NMR work with a $\mathrm{Cu}$ host, ${ }^{17}$ though in the latter case a saturation effect is apparent for high $\Delta Z$. Table VI shows that from $\Delta Z=1$ to $\Delta Z=2$, on the average the high frequencies double in value and for $A g \operatorname{Ir}(\Delta Z=3)$, the frequency is three times larger than that for $A g \operatorname{Pt}(\Delta Z=1)$.

\section{CONCLUSIONS}

The present results together with those of previous experiments show conclusively that for most of the alloys with impurities situated to the left of the host cubic metals $(\mathrm{Cu}, \mathrm{Ag}$, and $\mathrm{Au})$ in the Periodic Table there is a strong attraction between the probe In and the impurity atoms. This is in contrast to the situation for impurities to the right of the host metal Ag where repulsion was observed ${ }^{1}$ as discussed in Ref. 3 . The resulting large enhancement of the present impurity populations NN to the probe allowed us to study in detail different aspects of the impurity-probe interaction and of the EFG created by the impurity in the cubic host.

First we were able to follow in detail the onset of various $\mathrm{NN}$ configurations as the impurity concentration was increased. We have assumed that the lowest and first-appearing high frequency corresponds to a one NN impurity configuration. The other high frequencies appearing at higher concentrations were then successfully identified with configurations of more than one NN impurity on the basis of superpo- 
sition of EFG's, thus confirming our interpretation. This kind of investigation of the EFG generated by impurities of known concentration can be of importance in interpreting the results of similar experiments concerned with defects generated by radiation damage and by ion implantation in metals. Moreover, the TDPAC technique is a microscopic tool giving important local information at the damaged site, in contrast to the majority of the investigations of radiation damaged metals which have been performed by macroscopic techniques.

For those alloys where temperature effects were studied, the high-frequency interaction was found to have the usual empirical $T^{3 / 2}$ dependence in all cases, supporting the idea of lattice vibrations as the cause of the temperature dependence. Further support for this came from a correlation between the parameter $\alpha$, which gives the strength of the EFG temperature dependence, and the binding energy $\Delta E_{B}$. This gave $\alpha$ smaller for the more strongly bound probeimpurity pairs. For the low frequencies, generated by non-NN impurities, departures from the $T^{3 / 2}$ dependence in parts of the temperature range occurred for some alloys. This is difficult to analyze because as the temperature varied not only the low frequency changed, but also its distribution. However, where the $T^{3 / 2}$ dependence was followed the obtained $\alpha$ was an order of magnitude larger than for the high-

frequency interaction. This is expected as the binding between probe and non-NN impurities is much weaker than for $\mathrm{NN}$ impurities. It is interesting to compare our results with work on noncubic metals and on radiation damaged cubic metals. For ${ }^{111} \mathrm{Cd}$ in noncubic $s p$ metals, where a strong attraction between probe and host atoms is not expected, the $\alpha$ parameters are large; they are of the same order of magnitude as we observed for the low frequencies. On the other hand, the temperature dependence of the EFG created by vacancies in cubic systems ${ }^{18}$ is much weaker, giving $T^{3 / 2}$ slopes of the order of magnitude of those obtained for the NN high frequencies reported in the present paper.

The room-temperature high frequencies $\nu_{h}$ and the binding energies $\Delta E_{B}$ were analyzed on the basis of the differences in valence $(\Delta Z)$ and in period $(\lambda)$ between the impurity and the host. The measured binding energies were augmented by making estimates of $\Delta E_{B}$ for those alloys where temperature effects were not studied. A smooth dependence of both the binding energy and the high frequency on $\Delta Z$ and $\lambda$ was found. The dependence of $\nu_{h}$ on the period parameter $\lambda$ can be interpreted as an effect of the electronic core of both the impurity and the matrix. Very probably this correlation evidences an effect related to the number of inner electrons in the atomic core. The increase of $\nu_{h}$ with $\Delta Z$ is in line with previous work for impurities to the right-hand side of the matrix in the Periodic Table.
It is interesting to compare our results with those obtained from NMR experiments. Most of the NMR work has been done in $\mathrm{Cu}$ matrix. Nevald and Peter$\operatorname{sen}^{19}$ measured the EFG's created by $\mathrm{Ni}, \mathrm{Pd}$, and $\mathrm{Pt}$ impurities in a single-crystal matrix of $\mathrm{Cu}$. As discussed in Ref. 4 , our result for the EFG acting on Cd in the $C u \mathrm{Pd}$ alloy agrees very well with the value (also ascribed to one NN impurity) measured by NMR on $\mathrm{Cu}$ in the same alloy. ${ }^{19}$ In the alloy of $C u \mathrm{Pt}$, Nevald and Petersen have not succeeded to detect NMR lines due to NN impurity; they found a low frequency which was attributed to next NN impurities and which gives an EFG of $0.21 \times 10^{16} \mathrm{~V} / \mathrm{cm}^{2}$ after removing the probe dependence as usual by dividing by the Sternheimer factor. In the present experiment for $C u \mathrm{Pt}$ we observe very clearly a high frequency due to one NN impurity and a low distributed frequency due to more distant impurities (see Fig. 1 and Table II), from which we extract EFG's of $1.4 \times 10^{16}$ and $0.07 \times 10^{16} \mathrm{~V} / \mathrm{cm}^{2}$, respectively. Considering the agreement of the results for $C u \mathrm{Pd}$ it is difficult to understand why the EFG due to the NN $\mathrm{Pt}$ impurity was not observed in the NMR experiment, especially as the smaller-amplitude enhancement in $C u$ Pt would tend to make the TDPAC frequency determination, if anything less easy. Also our low distributed frequency is three times smaller than the NMR result, but one has to realize that the TDPAC technique is not able to resolve the contribution to the EFG from different non-NN shells.

In the present work we discuss our data from a phenomenological and systematic point of view, without a tentative interpretation of the EFG in terms of the existing models. The theoretical approach by Kohn and Vosko ${ }^{20}$ and Blandin and Friedel $^{21}$ were able to account semiquantitatively for the "wipeout numbers" deduced from NMR experiments, by calculating the EFG produced by longrange oscillatory screening charge cloud surrounding the impurity atom. The situation is different in the case of an EFG generated by NN impurity atoms. The above model is not suitable for this situation, since it is an asymptotic model. More recent calculations have also failed in reproducing the currently existing data. The model used by Fukai and Watanabe ${ }^{22}$ for Al-based alloys did not give the right order of magnitude, and calculations, performed for $\mathrm{Cu}$-based alloys, not only do not give the order of magnitude, but also do not follow the systematics of the experimental results. Recently Sagalyn and Alexander ${ }^{23}$ performed new calculations for the NMR data in $\mathrm{Cu}$-based alloys, using a model which combines the valence effect and the size effect. They obtain good fittings to the experimental results, concluding that the size effect is responsible for as much as $40 \%$ of the EFG in some alloys. The difficulty with this approach is that the used EFG-strain coupling constant required to account for the data is much larger 
than the constant inferred from experiments on plastically deformed pure Copper. It seems that we need more elaborate theoretical models in order to understand better the mechanism responsible for the EFG and also for the impurity-probe binding in doped cubic metals.

\section{ACKNOWLEDGMENTS}

This work was supported in part by Conselho $\mathrm{Na}$ cional de Desenvolvimento Científico e Tecnológico (CNPq) and Financiadora de Estudos e Projetos (FINEP).
"Present address: Comision Nacional de Energia Atómica, Buenos Aires, Argentina.

${ }^{+}$On leave from Universidad Nacional de La Plata and CONICET, Argentina, under the exchange program CNPqCONICET

IR. P. Livi, M. Behar, and F. C. Zawislak, Hyper. Inter. 5, 1 (1977).

${ }^{2}$ J. A. H. da Jornada, L. Amaral, I. J. R. Baumvol, M. Behar, R. P. Livi, and F. C. Zawislak, Phys. Lett. A $\underline{64}$, 246 (1977).

3J. A. H. da Jornada, I. J. R. Baumvol, M. Behar, R. P. Livi, and F. C. Zawislak, Hyper. Inter. 5, 219 (1978).

${ }^{4}$ I. J. R. Baumvol, M. Behar, J. A. H. da Jornada, E. R. Fraga, R. P. Livi, and F. C. Zawislak, J. Phys. F 9 , 1233 (1979).

${ }^{5} \mathrm{~K}$. Królas, K. Parliński, B. Wodniecka, and P. Wodniecki, Hyper. Inter. 4, 600 (1978).

${ }^{6} \mathrm{~K}$. Królas, B. Wodniecka, and P. Wodniecki, Hyper. Inter. 4, 605 (1978).

${ }^{7} \mathrm{M}$. Hansen, Constitution of Binary Alloys, 2nd ed. (McGrawHill, New York, 1958).

${ }^{8}$ R. P. Elliott, Constitution of Binary Alloys, 1st Suppl. (McGraw-Hill, New York, 1965).

${ }^{9}$ H. Frauenfelder and R. M. Steffen, in Alpha-, Beta- and
Gamma-Ray Spectroscopy, edited by K. Siegbahn (NorthHolland, Amsterdam, 1965), Vol. 2, p. 997.

$10 \mathrm{~J}$. Christiansen, P. Heubes, R. Keitel, W. Klinger, W. Loeffler, W. Sandner, and W. Witthuhn, Z. Phys. B 24, 177 (1976).

11 P. Jena, Phys. Rev. Lett. $\underline{36}, 418$ (1976).

${ }^{12}$ K. Nishiyama, F. Dimmling, Th. Kornrumpf, and D. Riegel, Phys. Rev. Lett. 37,357 (1976).

${ }^{13}$ M. D. Thompson, P. C. Pattnaik, and T. P. Das, Phys. Rev. B 18,5402 (1978)

${ }^{14}$ I. J. R. Baumvol, M. Behar, J. R. Iglesias-Sicardi, R. P. Livi, and F. C. Zawislak, Phys. Rev. B 18, 6713 (1978).

${ }^{15}$ J. R. Iglesias-Sicardi, Phys. Lett. A 64,406 (1978).

${ }^{16}$ A. O. E. Animalu, Phys. Rev. B $\underline{8}, 3542$ (1973).

${ }^{17}$ R. Nevald, B. L. Jensen, and P. B. Fynbo, J. Phys. F $\underline{4}$, 1320 (1974).

${ }^{18} \mathrm{~A}$. Weidinger, O. Echt, E. Recknagel, G. Schatz, and Th. Wichert, Phys. Lett. A $\underline{65}, 247$ (1978).

${ }^{19}$ R. Nevald and G. Petersen, J. Phys. F 5, 1778 (1975).

${ }^{20}$ W. Kohn and S. H. Vosko, Phys. Rev. 119,912 (1960).

${ }^{21}$ A. Blandin and J. Friedel, J. Phys. Radium 21, 689 (1960).

${ }^{22}$ Y. Fukai and K. Watanabe, Phys. Rev. B 10, 3015 (1974).

23P. L. Sagalyn and M. N. Alexander, Phys. Rev. B 15, 5581 (1977). 